\section{Capacidade de produção e grau de utilização de tomógrafo computadorizado no Sistema Único de Saúde}

\author{
CT scanners in the Brazilian Unified National \\ Health System: installed capacity \\ and utilization
}

\section{Capacidad de producción y grado de utilización del tomógrafo computarizado en el Sistema Único de Salud brasileño}

\author{
${ }^{1}$ Instituto de Saúde Coletiva, \\ Universidade Federal da \\ Bahia, Salvador, Brasil. \\ 2 Secretaria de Saúde do \\ Estado da Bahia, Salvador \\ Brasil. \\ 3 Instituto Federal de \\ Educação, Ciência e \\ Tecnologia da Bahia, \\ Salvador, Brasil. \\ Correspondência \\ D. L. Santos \\ Instituto de Saúde Coletiva \\ Universidade Federal da \\ Bahia. \\ Rua Basílio da Gama s/n, \\ Salvador, $B A$ \\ 40110-040, Brasil. \\ dianalima0203@gmail.com
}

\begin{abstract}
This study analyzes the supply and use of computed tomography scanners (CT) in the Brazilian Unified National Health System (SUS) according to State and administrative levels in the year 2009. Secondary data were used to estimate the installed CT capacity in public healthcare facilities and in private services outsourced by the SUS and calculated the rate of utilization. Average national CT utilization was less than $10 \%$. The public sector showed lower CT use than the private sector outsourced by the SUS. The number of CT tests in the SUS was less than half the number produced in developed countries. The results thus suggest the need for further studies on management practices with high-technology equipment in order to improve allocation of current and future public resources in supplying CT tests.
\end{abstract}

Material Resources in Health; State Health Care Coverage; Health Sciences, Technology and Innovation Management
Diana Lima dos Santos 1

Handerson Jorge Dourado Leite 2,3

Davide Rasella 1

Sebastião Antonio Loureiro de Souza e Silva ${ }^{1}$

\section{Resumo}

O objetivo deste estudo foi analisar a distribuição da oferta da tomografia computadorizada e o grau de utilização do tomógrafo computadorizado (TC) no Sistema Único de Saúde (SUS), por estado e esfera administrativa, no ano de 2009. Utilizando dados secundários, estimou-se a capacidade de produção dos tomógrafos públicose privados disponíveis ao SUS e calculou-se o grau de utilização dos mesmos. Os resultados mostraram que o grau de utilização dos TCs dos prestadores SUS possui uma média nacional abaixo de $10 \%$ e que o setor público tem o menor grau de utilização do TC, em comparação com o setor privado conveniado ao SUS. Foi observado que a produção de exames por TC no SUS é menor do que a metade da produção obtida em países desenvolvidos. Assim, os resultados sugerem a necessidade de um aprofundamento nos estudos das práticas de gestão tecnológica desses equipamentos, uma vez que é possível uma melhor alocação dos recursos públicos necessários à produção de tomografias computadorizadas.

Recursos Materiais em Saúde; Cobertura de Serviços Públicos de Saúde; Gestão de Ciência, Tecnologia e Inovação em Saúde 
O conhecimento sobre a capacidade produtiva tem importância estratégica para as organizações, uma vez que modificações no seu tamanho dependem de grandes investimentos de capital tecnológico e humano, sendo considerada como planejamento de longo prazo e com impactos diretos no desempenho operacional da unidade produtiva 1 . Do ponto de vista econômico, a unidade deve operar em um nível ótimo de produção, ou seja, quando o nível de produção consegue diluir ao máximo os custos fixos disponíveis ${ }^{2}$. Nesse sentido, um baixo grau de utilização da capacidade produtiva pode indicar uma subutilização dos recursos disponíveis, o que impacta negativamente nos custos, como também na oferta dos serviços.

Por capacidade de produção de um equipamento, entende-se como a máxima quantidade, em determinado período de tempo, que um equipamento pode produzir, sob condições normais de operação, levando em conta a jornada de trabalho que o estabelecimento adota, sem considerar qualquer tipo de perda, como as decorrentes de quebras do equipamento, manutenção preventiva ou corretiva, falta de funcionários, problemas de programação do equipamento, trocas de turnos, dentre outros ${ }^{3}$. Por sua vez, o grau de utilização, ou seja, a razão entre a produção real e a capacidade de produção do equipamento, é usada como uma medida fundamental de desempenho da produção, uma vez que pode indicar o quanto um equipamento está sendo utilizado, tendo por base a sua capacidade de produção ${ }^{3}$.

No campo da saúde e, especialmente no âmbito do Sistema Único de Saúde (SUS), o conhecimento de tais parâmetros pode auxiliar no mapeamento da capacidade de oferta dos serviços de saúde, impactando no acesso, na qualidade do atendimento e no planejamento para a aquisição e alocação de novas tecnologias de alto custo no sistema de saúde. Isso implica dizer que o uso dessas ferramentas tem grande relevância para a tomada de decisões estratégicas no âmbito das tecnologias em saúde.

A Programação Pactuada Integrada (PPI) 4, publicada em 2006, foi um instrumento desenvolvido pelo SUS para auxiliar na definição e quantificação das ações em saúde e compreende uma cobertura planejada dos serviços, conforme as necessidades da população local, buscando a equidade no acesso e a melhor alocação de recursos. Para orientar os gestores locais do SUS na construção da PPI, utilizou-se a Portaria MS/ GM no 1.101 5, de 2002, que define a necessidade de estabelecer parâmetros de cobertura e de produtividade assistenciais como instrumentos de planejamento, controle, regulação e avalia- ção do SUS. Para tanto, consideram-se necessárias, dentre outras ações, estimar a capacidade de produção e o grau de utilização dos recursos, equipamentos e serviços de assistência à saúde. As bases para cálculo desses parâmetros foram estabelecidas em 2004, no manual de auditoria na atenção básica 6 , com a finalidade de analisar a compatibilidade entre a capacidade de produção dos equipamentos e a produção realizada nas unidades de saúde.

Apesar da importância desses parâmetros, não foram encontradas publicações que apliquem esses conceitos para o campo das tecnologias médicas no Brasil. Estudos realizados no Canadá reconheceram a importância do uso da capacidade de produção e do grau de utilização do tomógrafo computadorizado (TC) como ferramentas necessárias para alocar e utilizar, de forma mais racional, essa tecnologia, visando ao melhor atendimento das necessidades da população 7,8. Os pesquisadores do Instituto Canadense de Informação em Saúde identificaram, por meio da aplicação dos conceitos de capacidade de produção e grau de utilização, que o uso do TC poderia ser mais bem otimizado e assim ampliar a produção de tomografias o suficiente para cobrir a demanda reprimida existente por estes exames.

O estudo de Ariste \& Fortin 8 constatou que entre as províncias do Canadá há diferenças no grau de utilização do TC; dados que confirmam os achados do estudo de Keller 7, também realizado no Canadá, que identificou que os TCs de 71 hospitais visitados na Província de Ontário estavam operando abaixo da capacidade de referência, ou seja, aquela que seria obtida caso os TCs operassem 16 horas por dia, 7 dias por semana. Com base nos resultados do estudo de Ariste \& Fortin 8, seria possível elevar, no Canadá, a capacidade de produção de exames para $68 \%$, sem aumentos adicionais em capital ou em infraestrutura.

Considerando que o SUS é o acesso à saúde de cerca de $80 \%$ da população brasileira (Departamento de Informática do SUS. Indicadores de Cobertura 2009. http://tabnet.datasus.gov.br/ cgi/idb2010/matriz.htm\#cober, acessado em 20/Mai/2012), o que nos motivou a realizar este estudo foram as informações encontradas em alguns trabalhos científicos e algumas notícias de caráter local, que sugerem escassez de oferta de tomografia computadorizada para a população assistida pelo SUS 9,10,11, que pode estar refletida, dentre outros indicadores, no longo tempo de espera dos pacientes para a realização destes exames 12,13,14,15,16. Isso pode indicar que a capacidade produtiva dos TCs é insuficiente para atender às demandas do SUS ou, ainda, que o grau de utilização dos equipamentos exis- 
tentes é baixo. Ou também, que existem menos TCs que o necessário para atender às demandas do SUS.

Assim, o objetivo deste estudo foi analisar a distribuição da capacidade de oferta de tomografia computadorizada e do grau de utilização do tomógrafo computadorizado, por Unidades da Federação (UF) e esferas pública e privada conveniadas ao SUS, no ano de 2009, comparando alguns resultados com os de outros países.

\section{Metodologia}

Trata-se de um estudo de agregados espaciais, que teve por unidade de análise a unidade federativa. Foram utilizados os dados de todas as UFs do Brasil para o ano de 2009. Escolhemos o ano de 2009 pois foi o ano mais recente em que os dados referentes ao número de tomógrafos estavam disponibilizados pelo Instituto Brasileiro de Geografia e Estatística (IBGE. Estatísticas da Saúde: Assistência Médico-Sanitária 2009. http://www.ibge.gov.br/home/, acessado em 20/ Mai/2012).

Para gerar o indicador de capacidade de pro$d u c ̧ a ̃ o$, foi necessário construir uma expressão matemática com base no conceito apresentado por Slack et al. 3. Tomou-se como referência a proposta para avaliar a capacidade de produção dos procedimentos nas unidades de saúde, conforme o manual de auditoria na atenção básica 6 . Assim, o indicador de capacidade de produção (CP), dado em quantidade de tomografias, foi construído como função do número de equipamentos, do tempo médio para a realização de cada exame e do tempo disponível do equipamento para a realização de exames em cada serviço.

Para obter o número de equipamentos de TC por cada prestador de serviço (público, privado conveniado e/ou total) e por UF, optou-se por utilizar uma combinação das bases de dados do Cadastro Nacional dos Estabelecimentos de Saúde (CNES; Recursos Físicos 2009. http:/ /www. datasus.gov.br, acessado em 20/Mai/2012) e do IBGE (Estatísticas da Saúde: Assistência MédicoSanitária 2009. http://www.ibge.gov.br/home/, acessado em 20/Mai/2012), referentes ao ano de 2009. Tal opção considerou que os dados sobre distribuição de TC disponibilizados pelo IBGE pareciam mais confiáveis do que os dados disponibilizados pelo CNES 9

O estudo de Gutierrez 9 identificou no banco de dados do CNES problemas no número de TC disponíveis ao SUS da rede pública, pois nem todos os estabelecimentos de saúde públicos informam os tomógrafos que disponibilizam ao SUS.
Além disso, confrontando os dados sobre número de TC fornecidos pelo CNES com os fornecidos pelo IBGE, identificaram-se, em níveis municipal e estadual, algumas variações no número de TCs. Essas incongruências de dados dificultariam a estimação dos dados, com base no número de tomógrafos apresentados no CNES, pois havia produção de exames em municípios que declaravam não possuir tomógrafos, e vice-versa.

Contudo, apesar da aparente maior confiabilidade na base de dados do IBGE, esta não apresentava detalhes sobre o tipo de estabelecimento em que se localizava cada TC e, portanto, não fornecia informações suficientes para identificar o tempo de funcionamento de cada tomógrafo que atende ao SUS, para cada UF.

Assim, como havia a necessidade de definir o número de TC existentes nos estabelecimentos de saúde de caráter ambulatorial e os que estavam localizados em serviços pré-hospitalares e hospitalares, recorreu-se aos dados disponibilizados pelo CNES, que fornece informações, por estado, sobre a natureza do estabelecimento que possui o TC, para os setores público e privado. Então, após excluir as distorções identificadas, foi calculado, com base nos dados da CNES, um percentual, por UF, dos estabelecimentos que funcionam 8 horas e 24 horas, e onde os TC estão localizados. Essa porcentagem obtida foi aplicada de forma estratificada ao número de TC existentes na UF, segundo o IBGE (Estatísticas da Saúde: Assistência Médico-Sanitária 2009. http://www. ibge.gov.br/home/, acessado em 20/Mai/2012), e agrupada por prestadores públicos e privados conveniados ao SUS, conforme a Tabela 1.

Dessa forma, identificou-se que em 2009, $4,9 \%$ dos estabelecimentos prestadores do SUS que funcionavam 8 horas eram públicos e 95,1\% eram privados. Já para os estabelecimentos prestadores do SUS que funcionavam 24 horas, verificou-se que $42 \%$ eram públicos e $58 \%$ privados.

A porcentagem obtida pelo CNES por estado foi multiplicada pelo número absoluto de equipamentos existentes em cada UF, segundo o IBGE (Estatísticas da Saúde: Assistência MédicoSanitária 2009. http://www.ibge.gov.br/home/, acessado em 20/Mai/2012), e agrupados por prestadores público e privado conveniados ao SUS.

O tempo médio para a realização de um exame tomográfico considerado foi de 30 minutos, conforme previsto em documentos práticos 17,18.

Para a definição do tempo disponível do equipamento para a realização de exames em cada serviço, convencionou-se que os equipamentos existentes nos estabelecimentos de caráter ambulatorial funcionam 8 horas/dia, distribuídos em dois turnos de 4 horas cada, durante 260 
Distribuição dos estabelecimentos que possuem tomógrafo computadorizado disponível ao Sistema Único de Saúde (SUS), por tipo de estabelecimento e horas diárias de funcionamento. Brasil, 2009.

\begin{tabular}{|c|c|c|c|}
\hline Tipo de estabelecimento & Público & Privado & Total \\
\hline Estabelecimentos ambulatoriais (8 horas) & 24 & 465 & 489 \\
\hline Centro de Atenção Psicossocial & 1 & 0 & 1 \\
\hline Centro de saúde/Unidade básica de saúde & 4 & 0 & 4 \\
\hline Clinica especializada/Ambulatório especializado & 10 & 114 & 124 \\
\hline Consultório & 0 & 6 & 6 \\
\hline Hospital-dia & 0 & 2 & 2 \\
\hline Policlínica & 6 & 22 & 28 \\
\hline Unidade de serviço de apoio de diagnose e terapia & 2 & 321 & 323 \\
\hline Unidade mista & 1 & 0 & 1 \\
\hline Estabelecimentos pré-hospitalares e hospitalares ( 24 horas) & 339 & 469 & 808 \\
\hline Hospital geral & 293 & 403 & 696 \\
\hline Hospital especializado & 28 & 63 & 91 \\
\hline Pronto socorro geral & 14 & 2 & 16 \\
\hline Pronto socorro especializado & 4 & 0 & 4 \\
\hline Unidade móvel de nível pré-hospitalar - urgência/emergência & 0 & 1 & 1 \\
\hline
\end{tabular}

Elaborado pelos autores com base no Cadastro Nacional dos Estabelecimentos de Saúde (CNES; Recursos Físicos 2009. http://www.datasus.gov.br, acessado em 20/Mai/2012).

dias por ano. Para os equipamentos pertencentes a estabelecimentos de caráter pré-hospitalar e hospitalar, considerou-se que funcionam 24 horas por dia, durante 365 dias/ano.

Assim, com base nas considerações realizadas, definiu-se a capacidade de produção como: $C P=\left[2080 T_{8}+8760 T_{24}\right] N_{T C} Z$ em que: $C P$ é a capacidade de produção, dada em número de exames/ano; $N_{T C}$ é o número de TC por estado; $T_{8}$ e $T_{24}$ são as taxas referentes aos equipamentos localizados em estabelecimentos que funcionam 8 horas e 24 horas, respectivamente. Os termos 8 horas e 24 horas referem-se ao total de horas de funcionamento dos estabelecimentos por dia, considerando que 2.080 e 8.760 fazem referência ao número de horas de funcionamento por ano, respectivo aos estabelecimentos de 8 e 24 horas; $Z$ representa o número médio de realização de exames produzidos, em número de exames/hora. Foi adotada a média de 2 exames por hora.

A capacidade de produção do TC pela população foi obtida por meio da razão entre as capacidades de produção dos prestadores público, privado conveniado ao SUS e total SUS, e a população residente nos estados, que foi obtida pelas projeções populacionais estimadas pelo IBGE, disponíveis na página de Internet do Departamento de Informática do SUS (DATASUS. Informações Demográficas - População Residente
- 2009. http://www.datasus.gov.br, acessado em 20/Mai/2012), referente ao ano de 2009.

O indicador grau de utilização do TC foi obtido pelo resultado da produção de tomografias realizadas no SUS durante o ano de 2009, considerando cada prestador (público, privado conveniado ao SUS) dividido pelas suas respectivas capacidades de produção. O grau de utilização total SUS é resultante da razão entre a produção de tomografias realizadas no SUS e a soma das capacidades de produção pública e privada conveniada ao SUS. Os dados sobre produção de tomografias realizadas por local de atendimento, entre janeiro e dezembro de 2009, foram obtidos do DATASUS (Produção Ambulatorial, por Local de Atendimento - 2009. http://www.datasus. gov.br, acessado em 20/Mai/2012), identificados pelos procedimentos do subgrupo 0206, que compreende todos os procedimentos de diagnóstico por tomografia computadorizada, financiados pelo SUS.

$G U[\%]=100 P / C P$

em que: $G U$ é o grau de utilização, dado em porcentual; $P$ é a produção de exames realizada pelo SUS, dada em número de exames por ano; $C P$ é a capacidade de produção do equipamento, dada em número de exames.

Os resultados de todos os indicadores capacidade de produção e grau de utilização foram apresentados para cada UF e em médias regio- 
nais, que foram calculadas com base no somatório dos dados dos estados de cada região, divididos pelo número de estados de cada região.

Gerou-se também uma média nacional do número de tomografias produzidas no SUS por habitantes e outra média nacional de número de tomografias produzidas no SUS por número de tomógrafos computadorizados, por cada prestador do SUS, para comparação com as médias de outros países, publicadas nas estatísticas da Organização para a Cooperação e Desenvolvimento Económico (OCDE; Statistics. http://stats.oecd. org /index.aspx? DataSetCode=HEALTH_STAT, acessado em 17/Mar/2013). Para o primeiro, tomou-se a produção de tomografias (de cada prestador SUS) dividida pelo número de habitantes. Para o segundo indicador, buscando-se identificar a produção média de tomografias SUS por TC, dividiu-se a produção de tomografias pelo número de TCs, para cada prestador SUS.

\section{Resultados}

Na Tabela 2, são apresentadas as informações sobre a capacidade de produção dos TCs disponíveis ao SUS por habitantes, bem como o grau de utilização do TC no âmbito do SUS. A Figura 1, ilustrando e complementando as informações da Tabela 2, apresenta a capacidade de produção de cada prestador, bem como o total dos prestadores do SUS.

A capacidade de produção nacional média do TC no SUS foi de 120,6 tomografias para cada 1.000 habitantes. As regiões com as maiores capacidades de produção SUS foram Centrooeste $(234,9)$ Sudeste $(162,0)$ e Sul $(151,8)$. As regiões Norte e Nordeste tiveram capacidades de produção total SUS per capita abaixo da média nacional.

O setor privado conveniado ao SUS apresentou uma capacidade de produção nacional média de 90,2 tomografias por cada 1.000 habitantes, sendo a maior e menor médias verificadas, respectivamente, nas regiões Centro-oeste $(191,1)$ e Norte $(34,9)$. O Norte e o Nordeste obtiveram capacidade de produção do TC privado abaixo da média nacional para quase todos os estados, com exceção apenas de Rondônia, que apresentou uma capacidade de produção de 95,5 tomografias para cada 1.000 habitantes.

A capacidade de produção pública média do Brasil foi de 30,4 tomografias para cada 1.000 habitantes, o que representa cerca de $25 \%$ do total ofertado pelo SUS, considerando a capacidade de produção total do SUS. Roraima e Amapá possuem as maiores capacidades de produção pública do SUS (83,1 e 83,9 respectivamente), o que contribuiu para que o Norte registrasse a maior capacidade de produção de tomografia pública do Brasil (45,5 exames para cada 1.000 habitante). O Rio de Janeiro apresentou a maior capacidade de produção de tomografias do Sudeste $(58,4)$ e a Região Centro-oeste foi a que registrou a segunda maior capacidade de produção de tomografia pelo setor público (43,8 exames para cada 1.000 habitante).

A Tabela 2 também apresenta informações regionais sobre o grau de utilização do TC, considerando que cada prestador apresenta a relação entre a capacidade de produção e a produção realizada para o SUS. A Figura 2 ilustra nos mapas o grau de utilização para cada prestador (público e privado conveniado ao SUS), bem como o total SUS, considerando a soma das capacidades de produção do setor privado conveniado ao SUS e do setor público. As regiões Sul e Nordeste apresentaram as mais baixas capacidades de produção de tomografias pelo setor público (15,4 e 17,6 exames para cada 1.000 habitantes, respectivamente).

Identificou-se que o grau de utilização médio do TC no SUS foi de 9,4\%, considerando a razão entre a produção de tomografias totais pelo SUS e a capacidade de produção total do TC no SUS. As regiões Norte e Nordeste apresentam as maiores médias de grau de utilização total do TC (14\%, e $10,8 \%$, respectivamente) e o Centrooeste registra a menor média $(4 \%)$.

Considerando ainda o grau de utilização brasileiro do TC no SUS, observou-se que os estados que estão abaixo da média nacional concentraram-se predominantemente no Centro-oeste e Sul, ocorrendo o mesmo em cinco estados do Nordeste, três no Norte e um no Sudeste. O setor privado utilizou a média nacional de $15 \%$ de sua capacidade de produção para o SUS, com um significativo destaque para a Região Norte, cujo grau de utilização foi de $39,2 \%$, verificando-se em Roraima e Amapá graus de utilização do setor privado para o SUS, respectivamente de $145,8 \%$ e 94,24\%. O menor grau de utilização do setor privado conveniado ao SUS foi verificado nas regiões Centro-oeste $(2,7 \%)$, Sudeste $(4,4 \%)$ e Sul $(4,9 \%)$. O setor público utilizou em média $14 \%$ de sua capacidade de produção de tomografias, com apenas 11 estados ultrapassando esta média, porém não excedendo a $35 \%$ de capacidade de produção em cada estado, com exceção do Ceará, que utilizou $37,6 \%$ de sua capacidade de produção. A região com o maior grau de utilização do setor público é o Sudeste (17,9\%), seguida da Região Sul (15,7\%) e do Nordeste $(15,1 \%)$. A média dos graus de produção públicos das demais regiões está abaixo da média nacional. 
Tabela 2

Capacidade de produção * e grau de utilização do tomógrafo computadorizado (TC) no Sistema Único de Saúde (SUS). Média das Unidades Federativas (UF) e regiões do Brasil, 2009.

\begin{tabular}{|c|c|c|c|c|c|c|}
\hline \multirow[t]{2}{*}{ UF } & \multicolumn{3}{|c|}{ Capacidade de produção do TC (por 1.000 habitantes) } & \multicolumn{3}{|c|}{ Grau de utilização do TC (\%) } \\
\hline & Total SUS & Privado (SUS) ** & Pública & Total SUS & Privado (SUS) ** & Público \\
\hline Norte (média) & 80,5 & 34,9 & 45,5 & 14,00 & 39,20 & 11,80 \\
\hline Rondônia & 118,8 & 95,5 & 23,3 & 3,97 & 4,94 & 0,00 \\
\hline Acre & 50,7 & - & 50,7 & 26,61 & 0,00 & 26,61 \\
\hline Amazonas & 69,0 & 32,9 & 36,1 & 9,42 & 14,31 & 4,97 \\
\hline Roraima & 93,0 & 9,9 & 83,1 & 18,09 & 145,89 & 2,92 \\
\hline Pará & 81,9 & 53,7 & 28,2 & 8,79 & 6,67 & 12,84 \\
\hline Amapá & 97,2 & 13,3 & 83,9 & 17,46 & 94,24 & 5,31 \\
\hline Tocantins & 52,9 & 39,3 & 13,6 & 13,94 & 8,37 & 30,09 \\
\hline Nordeste (média) & 72,4 & 54,8 & 17,6 & 10,80 & 9,80 & 15,10 \\
\hline Maranhão & 42,5 & 23,2 & 19,3 & 25,90 & 27,10 & 24,46 \\
\hline Piauí & 86,2 & 63,9 & 22,3 & 9,21 & 8,71 & 10,63 \\
\hline Ceará & 78,0 & 63,0 & 15,0 & 11,66 & 5,48 & 37,60 \\
\hline Rio Grande do Norte & 87,1 & 64,7 & 22,3 & 7,90 & 9,27 & 3,94 \\
\hline Paraíba & 105,3 & 86,7 & 18,6 & 4,51 & 4,61 & 4,05 \\
\hline Pernambuco & 49,0 & 35,1 & 13,9 & 15,31 & 17,41 & 10,01 \\
\hline Alagoas & 63,8 & 47,2 & 16,7 & 7,15 & 7,88 & 5,06 \\
\hline Sergipe & 64,7 & 47,4 & 17,3 & 7,82 & 3,68 & 19,10 \\
\hline Bahia & 74,6 & 62,0 & 12,6 & 7,35 & 4,48 & 21,44 \\
\hline Sudeste (média) & 162,0 & 131,2 & 30,7 & 6,50 & 4,40 & 17,90 \\
\hline Minas Gerais & 134,7 & 116,3 & 18,4 & 5,50 & 4,63 & 11,02 \\
\hline Espírito Santo & 155,5 & 138,0 & 17,5 & 6,91 & 4,90 & 22,75 \\
\hline Rio de Janeiro & 188,0 & 129,6 & 58,4 & 3,87 & 3,23 & 5,31 \\
\hline São Paulo & 169,6 & 141,1 & 28,6 & 9,59 & 4,94 & 32,57 \\
\hline Sul (média) & 151,8 & 136,4 & 15,4 & 5,90 & 4,90 & 15,70 \\
\hline Paraná & 134,7 & 123,1 & 11,7 & 5,18 & 4,78 & 9,36 \\
\hline Santa Catarina & 145,1 & 126,1 & 19,0 & 5,62 & 5,50 & 6,45 \\
\hline Rio Grande do Sul & 175,4 & 160,0 & 15,4 & 6,81 & 4,46 & 31,20 \\
\hline Centro-oeste (média) & 234,9 & 191,1 & 43,8 & 4,0 & 2,70 & 10,40 \\
\hline Mato Grosso do Sul & 357,4 & 298,0 & 59,4 & 1,32 & 0,93 & 3,25 \\
\hline Mato Grosso & 127,0 & 97,8 & 29,2 & 5,26 & 5,10 & 5,78 \\
\hline Goiás & 157,0 & 133,3 & 23,7 & 5,42 & 2,91 & 19,56 \\
\hline Distrito Federal & 298,2 & 235,2 & 62,9 & 4,19 & 1,82 & 13,07 \\
\hline Brasil (média) & 120,6 & 90,2 & 30,4 & 9,40 & 15,00 & 14,00 \\
\hline
\end{tabular}

* Considerando a soma das capacidades de produção dos tomógrafos públicos e privados conveniados ao SUS;

** Privado conveniado ao SUS.

Observou-se, por fim, que para a produção de tomografias realizadas no SUS o grau de utilização do TC do setor público (14\%) foi inferior ao verificado para o setor privado conveniado ao SUS (15\%), no Brasil.

A Tabela 3 apresenta uma comparação entre o Brasil e alguns países desenvolvidos e em desenvolvimento quanto ao número médio de exames de tomografia computadorizada para cada 1.000 habitantes, e o número médio de tomografias re- alizadas para cada TC em 2009. Identificou-se que os países desenvolvidos produziram em média mais de 100 tomografias para cada 1.000 habitantes. O Chile produziu 50,2 tomografias para cada 1.000 habitantes, e o Brasil produziu uma média de 8,8 tomografias para o mesmo montante populacional. Identificou-se também que os países mais desenvolvidos produziram mais tomografias por cada TC, verificando-se valores como 2.365 exames por TC na Austrália e mais de 8.000 
Capacidade de produção do tomógrafo computadorizado disponível ao Sistema Único de Saúde (SUS) para cada 1.000 habitantes, por tipo de prestador e total SUS, em cada Unidade Federativa. Brasil, 2009.

1a) Capacidade de produção total do SUS

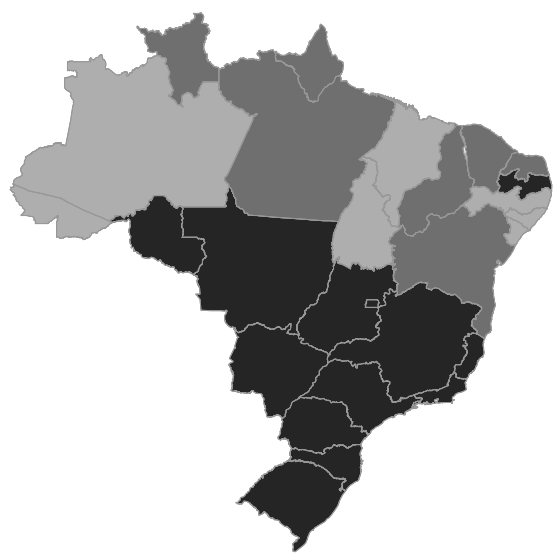

1b) Capacidade de produção total

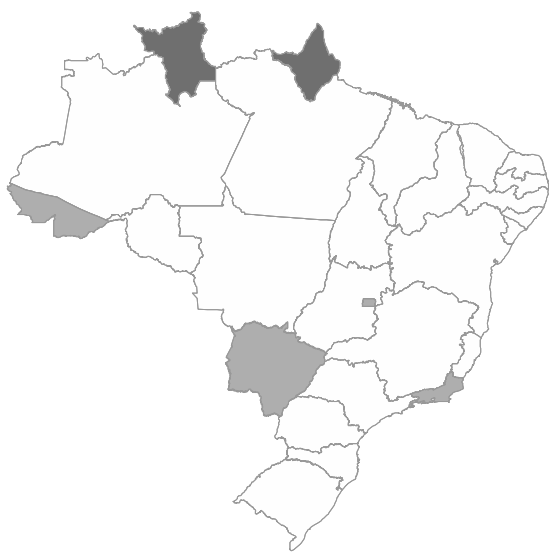

1c) Capacidade de produção privada

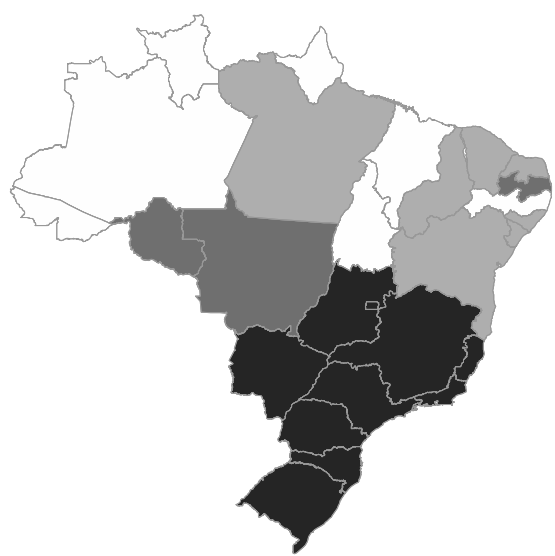

Exames/1.000 habitantes

abaixo de 40

40 a 70

70 a 100

acima de 100

exames por TC em países como Canadá, França e Israel. No Brasil, a produção média para o SUS em 2009 foi de 1.530 tomografias para cada TC.

\section{Discussão}

Este trabalho buscou estimar a capacidade de produção do TC no SUS e seu o grau de utilização, no ano de 2009.

Estudos realizados no Canadá investigaram os determinantes da oferta insuficiente de diagnósticos por imagem no país, a exemplo da tomografia computadorizada 7,8,19. Ariste \& Fortin 8 identificaram possíveis explicações para as diferenças do grau de uso do TC verificadas nas províncias do Canadá, como diferenças na densidade populacional, variações nas necessidades de saúde de cada local, ou no sistema de pagamentos dos serviços de saúde entre os locais. Outros estudos realizados no Canadá 7,19 concluíram que deficiências no financiamento local dos serviços de saúde, a falta de profissionais de radiologia, considerando os técnicos radiologistas e os técnicos responsáveis por manutenções corretivas e preventivas, e a inadequada gestão dos serviços ofertados para a população têm impedido a expansão dos serviços de diagnóstico por tomografia computadorizada.

No Brasil, a oferta pública de serviços de saúde é realizada pelo SUS, cuja parte significativa dos procedimentos de diagnóstico por imagem, dentre outros serviços ofertados por este sistema, é suprida por instituições privadas, em complemento aos serviços realizados pelo prestador público de saúde 20 .

Isso é possível porque a Lei Federal no 8.080/ 90 21, que institui o SUS, em seu Art. 4o, assegura a participação da assistência privada de forma complementar ao SUS, por meio de convênios e contratos. Ou seja, a Constituição Federal, em seu Art. 196, assegura que o setor privado deve ser prestador de serviços de saúde ao SUS quando a capacidade de produção do estado for insuficiente para atender à demanda 22 . Isso, aliado a outros fatores 20 , permitiu ao setor privado se 
2a) Grau de utilização total do SUS

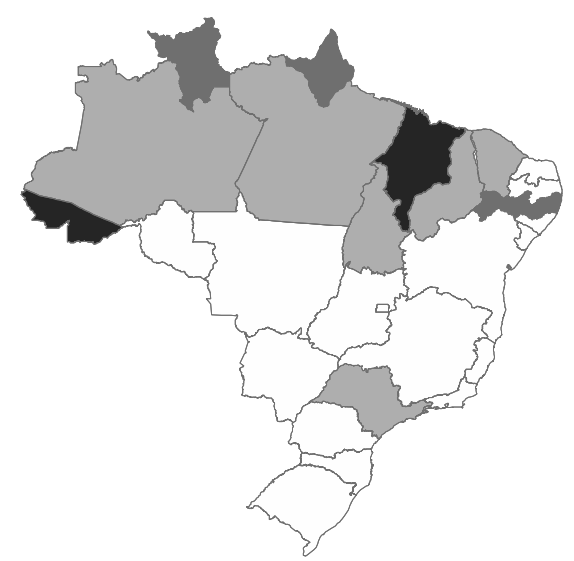

2b) Grau de utilização público

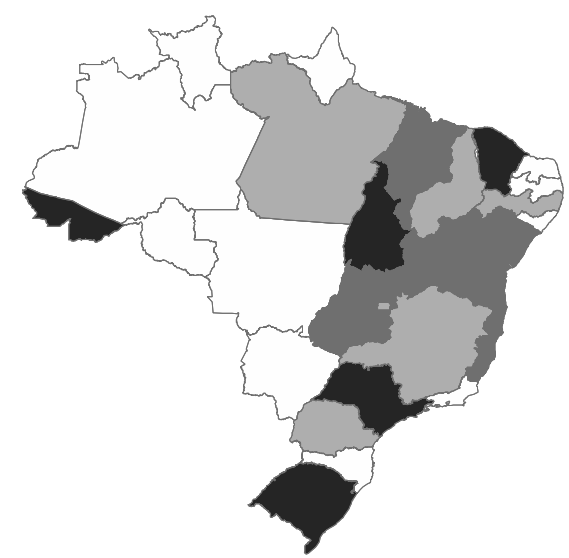

2c) Grau de utilização privado, conveniado com o SUS

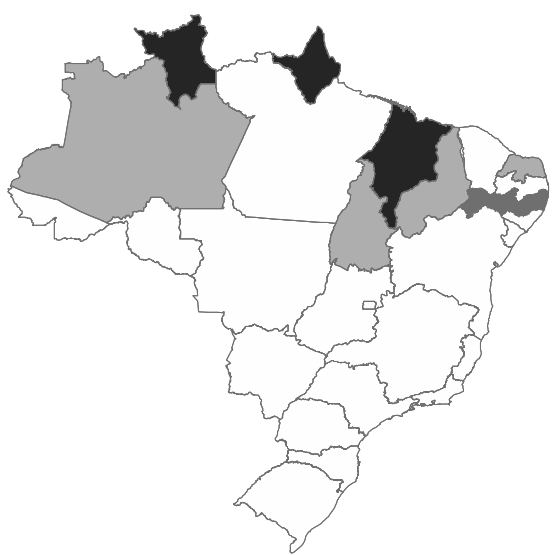

Grau de utilização (\%)

$\square$ abaixo de 8

8 a 15

15 a 25

acima de 25 organizar de forma dominante na oferta de procedimentos de maior lucratividade, como os de diagnóstico por imagem 10,20,22.

Observou-se que o grau de utilização do TC do setor privado conveniado para o SUS é superior ao grau de utilização do TC público, mesmo levando-se em conta que seja questionável o uso do setor privado para a oferta de TC ao SUS. Ou seja, o grau de utilização do TC privado é proporcionalmente maior nos estados e regiões onde é menor o grau de utilização do TC público, mesmo em regiões e estados onde o setor público tem uma maior potencialidade de uso da capacidade de produção de seus TCs.

Tal cenário pode ser resultante de uma gestão pública da tecnologia pouco eficiente, resultando na aquisição de equipamento que não é operado por falta de profissional capacitado, paradas prolongadas e excessivas para as manutenções preventivas e corretivas e, ainda, problemas no suprimento de eletricidade da unidade ou da região. Deve-se ter em conta, por fim, que é possível que o setor privado tenha uma preocupação maior com a estrutura de custos referentes à aqui- sição de equipamentos como TC. Dessa forma, é plausível uma maior pressão, por esse prestador, para a realização de exames de tomografia, com a finalidade de cobrir, com os exames realizados, os custos referentes ao investimento feito.

Foi possível também observar diferenças no grau de utilização entre as distintas regiões e UFs. Deve-se considerar nesse cenário as mesmas hipóteses levantadas para as variações entre o grau de utilização do setor privado e do setor público, acrescentando-se o argumento de que, em locais onde observou-se uma maior capacidade de produção per capita, houve consequentemente um menor grau de utilização de TC. Assim, é possível que a maior oferta pulverize a procura, resultando em menor uso relativo dessa tecnologia.

Uma atenção maior deve ser dada à Região Norte, onde o setor privado conveniado ao SUS utilizou cerca de três vezes mais o TC para o SUS que o setor público (ainda que os TCs desta região tenham utilizado apenas $11,8 \%$ da sua capacidade de produção). Verificaram-se inclusive estados onde há uma produção de tomografias realizadas pelo setor privado acima de $90 \%$ da 
Tabela 3

Exames de tomografia por 1.000 habitantes e por tomógrafo computadorizado (TC). Média dos países, 2009.

\begin{tabular}{lcc}
\hline Países & Exames (por 1.000 habitantes) & Exames por TC * \\
\hline Brasil & 8,8 & $1.317,4$ \\
Austrália & 91,5 & $2.365,2$ \\
Coreia do Sul & 92,6 & $2.517,2$ \\
Dinamarca & 91,5 & $3.855,8$ \\
Islândia & 156,2 & $4.531,9$ \\
Países Baixos & 65,2 & $5.795,4$ \\
República Tcheca & 87,5 & $6.202,7$ \\
Eslováquia & 85,4 & $6.425,6$ \\
Luxemburgo & 189,6 & $6.864,5$ \\
Turquia & 96,3 & $8.282,1$ \\
Canadá & 122,2 & $8.884,0$ \\
Estônia & 152,7 & $10.235,0$ \\
França & 138,3 & $12.482,8$ \\
Israel & 122,8 & $13.126,2$ \\
Chile & 50,2 & $* *$ \\
Estados Unidos & 252,7 & $* *$ \\
\hline Adaptado pes
\end{tabular}

Adaptado pelos autores com base em dados da Organização para a Cooperação e Desenvolvimento Econômico (OCDE Health Data 2012; http://stats.oecd.org/, acessado em 21/Mar/2013).

* Para o Brasil, trata-se da relação entre número de exames de tomografia SUS por número de TCs disponíveis ao SUS

(público mais privado conveniado ao SUS);

** Dados não apresentados pelos países listados para o ano de 2009.

sua capacidade de produção. Uma explicação para o grau de uso do TC privado acima de $100 \%$ seria que os prestadores privados não conveniados também pudessem ter prestado serviços ao SUS no ano de 2009, embora este fenômeno não se justifique, ante o baixo grau de utilização do TC do setor público quando comparado ao setor privado conveniado ao SUS.

De acordo com os dados apresentados, caso o setor público realizasse toda a produção de tomografias SUS em 2009, ele estaria utilizando cerca de $40 \%$ de sua capacidade de produção, o que implica dizer que possivelmente ainda estaria operando com capacidade ociosa.

Outra observação importante a se fazer quanto à utilização do TC consiste no tempo de funcionamento dos estabelecimentos onde eles estão alocados. De acordo com dados do CNES, apresentados na Tabela 1, cerca de $88 \%$ dos TCs públicos estão locados em estabelecimentos 24 horas, e no setor privado conveniado ao SUS este número está em torno de $61 \%$. Mesmo considerando que os TCs públicos têm mais tempo disponível para operação que os TCs privados, os TCs públicos apresentaram aproximadamente metade do grau de utilização verificado nos TCs privados conveniados ao SUS.
Analisando os dados da Tabela 3, que compara a média brasileira de produção de tomografias realizadas pelo SUS para cada 1.000 habitantes e para cada TC com os resultados de alguns países publicados nas estatísticas da OCDE, notou-se que o Brasil realizou cerca de dez vezes menos tomografias para cada 1.000 habitantes, em comparação com estes países (os Estados Unidos realizam cerca de 20 vezes mais tomografias por 1.000 habitantes que o Brasil).

Quanto à produção de tomografias por TC, observou-se que o Brasil produziu menos de 1.000 tomografias SUS por cada TC quando comparado com países como Austrália ou Coreia do Sul. O Canadá produziu cerca de quatro vezes mais tomografias por TC que o Brasil, e países como Israel e França produziram pelo menos oito vezes mais tomografias por TC que o nosso país.

A análise dos dados da Tabela 3 pode indicar dois caminhos: ou há um excesso de produção de tomografias computadorizadas por população nos países listados anteriormente, ou a produção de tomografias per capita realizadas no Brasil está muito abaixo das reais necessidades da população local. 


\section{Considerações finais}

Os resultados do presente estudo identificaram o baixo grau de utilização do TC e a variabilidade deste indicador entre os estados e regiões no Brasil. Indicou também uma capacidade ociosa do TC público de $86 \%$ e de cerca de $90 \%$ para todos os TCs disponíveis ao SUS. Também verificouse que a produção de exames por TC no Brasil é muito baixa, quando comparada com outros países.

Nesse sentido, os resultados sugerem um aprofundamento nas práticas de gestão tecnológica dos TCs pertencentes ao setor público, uma vez que pode ser possível uma alocação mais racional dos recursos públicos necessários à produção de exames de tomografias computadorizadas. Com base nos indicadores desenvolvidos neste trabalho, é possível aos gestores do SUS avaliar os desempenhos dos TCs existentes nos diferentes tipos de unidades de saúde e nas diferentes localidades.

O estudo sugere também que é possível a ampliação da oferta do SUS para exames de tomografia computadorizada para a população, se provada a sua necessidade, sem que sejam necessários, em princípio, novos investimentos públicos em infraestrutura ou mais gastos com pagamentos de serviços privados de tomografias. Isso indica que uma melhor gestão dos equipamentos públicos existentes de TC pode promover uma oferta mais racional de exames de tomografias pelo SUS, otimizando os recursos existentes e reduzindo potenciais gastos desnecessários com aquisição privada destes serviços.

Os limites deste estudo consistem na dificuldade encontrada para a criação dos indicadores de capacidade de produção e do grau de utilização do TC, resultantes da inexatidão do banco de dados do CNES. Tais dificuldades geraram a necessidade de uso do banco de dados do IBGE para a formação das variáveis de capacidade de produção do TC e grau de produção, o que pode não ter gerado valores exatos para estes indicadores. Isso sugere que é necessário promover uma melhora no banco de dados do CNES, uma vez que são dados importantes pela frequência da atualização de dados e também pelo grau de detalhamento das informações, quando comparado aos dados do IBGE para este tema. Dados oficiais referentes à demanda reprimida por cada UF também poderiam fortalecer a validação dos resultados apresentados no presente trabalho.

É importante também comentar que verificou-se uma produção de tomografias acima da capacidade de produção dos TCs disponíveis ao SUS para alguns estados, fato que merece ser melhor investigado. É também possível a sub- notificação dos serviços de tomografia computadorizada, uma vez que observou-se, com base em dados do CNES e da produção ambulatorial do DATASUS, a existência de TCs em locais onde não houve produção de exames para o ano estudado.

A ausência de informações sobre a produção de tomografias realizadas pelo setor privado não conveniado ao SUS impediu uma análise mais ampla e detalhada sobre o grau de utilização do tomógrafo computadorizado no sistema de saúde brasileiro, o que limitou o presente estudo à produção SUS de tomografias computadorizadas. Por fim, o desconhecimento quanto ao número de tomografias computadorizadas que são destinadas ao SUS por cada prestador privado apresenta uma grande limitação deste estudo, de forma que não foi possível fornecer um valor real do grau de utilização do TC privado conveniado ao SUS, ou seja, este valor foi super ou subestimado em relação à capacidade de produção real do setor privado disponibilizado ao SUS.

Não foram encontrados estudos anteriores realizados no Brasil sobre análise dos indicadores de desempenho aqui aplicados. Por conta disso, utilizou-se como comparador estudos desenvolvidos no Canadá, que foram as únicas publicações encontradas sobre o tema. Deve-se compreender a relevância da utilização de tais estudos pelo fato de tratar de um país com um sistema de saúde universal, e que possui importantes organismos de avaliação de tecnologias, que atuam de forma articulada.

As limitações encontradas neste trabalho apontam as dificuldades de se implementar de forma mais ampla a Política Nacional de Gestão de Tecnologias em Saúde no SUS, tanto em termos de homogeneização das práticas existentes nas diferentes localidades, como também em termos da necessidades de ampliação das ferramentas de gestão das tecnologias em saúde, e também em termos de melhorar as bases de dados secundários para promover análises mais condizentes com a realidade.

Não foi objetivo deste estudo investigar os efeitos econômicos do grau de utilização do TC no SUS, como, por exemplo, o impacto nos custos, o que poderá ser realizado em trabalhos futuros.

Considerando a importância do uso dos indicadores de gestão do equipamento aqui estudado, recomenda-se que trabalhos futuros possam aplicá-lo em outras tecnologias médicas, para auxiliar na alocação mais racional dos recursos tecnológicos no SUS. 


\section{Resumen}

El objetivo de este estudio fue analizar la distribución de la oferta de tomografía computarizada y el grado de utilización del tomógrafo computarizado (TC) en el Sistema Único de Salud (SUS) brasileño, en los diferentes estados y niveles administrativos, durante 2009. Se utilizaron datos secundarios, se evaluó la capacidad de producción de tomógrafos públicos y privados, disponibles para el SUS, y se calculó el grado de utilización de los mismos. Los resultados mostraron que la utilización de los tomógrafos contratados por el SUS tienen un promedio nacional menor al 10\% y que el sector público tiene un menor grado de utilización del TC, en comparación con el sector privado contratado por el SUS. Se observó que la producción de exámenes por TC en el SUS es menor que la mitad de la producción observada en los países desarrollados. De esta forma, los resultados sugieren la necesidad de profundizar en las investigaciones sobre prácticas de gestión de esos equipos, ya que puede ser posible una mejor utilización de los recursos públicos necesarios para la producción de tomografías computarizadas.

Recursos Materiales en Salud; Cobertura de Servicios Públicos de Salud; Gestión de Ciencia, Tecnología e Innovación en Salud

\section{Colaboradores}

D. L. Santos participou da concepção e projeto, análise e interpretação dos dados, redação do artigo, revisão crítica relevante do conteúdo intelectual e aprovação final da versão a ser publicada. H. J. D. Leite participou da concepção e projeto, revisão crítica relevante do conteúdo intelectual e aprovação final da versão a ser publicada. D. Rasella participou da análise e interpretação dos dados e revisão crítica relevante do conteúdo intelectual. S. A. L. S. Silva participou da análise e interpretação dos dados, revisão crítica relevante do conteúdo intelectual e aprovação final da versão a ser publicada.

\section{Agradecimentos}

À FAPESB pelo apoio financeiro. Aos professores Luis Eugenio Portela Fernandes e Marcus Vinicius Teixeira Navarro, pelo auxílio científico. Este trabalho integra o INCT-Citecs, projeto financiado pelo Programa Institutos Nacionais de Ciência e Tecnologia (MCT-CNPq; processo oㅗ 57386/2008-9).

\section{Referências}

1. Correa HL, Corrêa CA. Administração de produção e de operações - manufatura e serviços: uma abordagem estratégica. São Paulo: Editora Atlas; 2005.

2. Pindyck RS, Rubinfeld DL. Microeconomia. $5 \underline{a}$ Ed. São Paulo: Prentice Hall; 2002.

3. Slack N, Chambers S, Harland C, Harrison A, Johnson R. Administração da produção. São Paulo: Editora Atlas; 1999.

4. Departamento de Regulação, Avaliação e Controle de Sistemas, Secretaria de Atenção à Saúde, Ministério da Saúde. Diretrizes para a programação pactuada e integrada da assistência à saúde. Brasília: Ministério da Saúde; 2006.

5. Ministério da Saúde. Portaria GM/MS no 1.101, de 12 de junho de 2002. Estabelece os parâmetros de cobertura assistencial no âmbito do Sistema Único de Saúde - SUS. Diário Oficial da União 2002; 13 jun.
6. Departamento Nacional de Auditoria do SUS, Ministério da Saúde. Orientações para proceder auditoria na Atenção Básica. Brasília: Ministério da Saúde; 2004.

7. Keller A. Ontario's wait time strategy. MRI and CT EXPERT PANEL PHASE I Report. https://ospace. scholarsportal.info/handle/1873/3151 (acessado em 06/Jun/2007).

8. Ariste R, Fortin G. Could MRI and CT scanners be operated more intensively in Canada? Healthc Policy 2007; 3:e113-20.

9. Gutierrez M. A oferta de tomógrafo computadorizado para o tratamento do acidente vascular cerebral agudo, no Brasil, sob o ponto de vista das desigualdades sociais e geográficas [Dissertação de Mestrado]. Rio de Janeiro: Escola Nacional de Saúde Pública Sergio Arouca, Fundação Oswaldo Cruz; 2009. 
10. Pessoto UC, Heimann LS, Boaretto RC, Castro IEN, Kayano J, Ibanhes LC, et al. Desigualdades no acesso e utilização dos serviços de saúde da Região Metropolitana de São Paulo. Ciênc Saúde Coletiva 2007; 12:351-62.

11. Confederação Nacional dos Municípios. Pesquisa da CNM sobre a demanda reprimida em saúde no Estado do Rio Grande do Sul - Brasília, 2011. http://www.cnm.org.br/ (acessado em 20/Nov/ 2012).

12. Machado G. Estratégia de equidade - um estudo da acessibilidade aos exames de média e alta complexidade no SUS - Goiás [Dissertação de Mestrado]. Goiânia: Universidade Católica de Goiás; 2006.

13. Viacava F, Ugá MAD, Porto S, Lagardia J, Moreira RS. Avaliação de desempenho de sistemas de saúde: um modelo de análise. Ciênc Saúde Coletiva 2012; 17:921-34.

14. Azevedo MCCV. Identificação de crianças e adolescentes com suspeita de câncer: uma proposta de intervenção [Dissertação de Mestrado]. Natal: Universidade Federal do Rio Grande do Norte; 2010.

15. Silva FO. Racionalização de gastos: uma abordagem discursiva da diretriz que ameaça o SUS [Monografia de Especialização]. Recife: Centro de Pesquisas Aggeu Magalhães, Fundação Oswaldo Cruz; 2007.
16. Sobreira S. Anos de espera para fazer exame. Jornal de Brasília 2012; 17 ago.

17. Fenelon S. Tomografia computadorizada. http:// www.imaginologia.com.br (acessado em 21/ Out/2012).

18. Vieira M. Análise económica da prestação de serviços de diagnóstico por imagem: TAC e ressonância magnética. http://www.fns.pt/docs/tac.pdf (acessado em 06/Nov/2012).

19. Emery DJ, Forster AJ, Shojania KG, Magnan S, Tubman M, Feasby TE. Management of MRI wait lists in Canada. Healthc Policy 2009; 4:76-86.

20. Paim JS, Travassos C, Almeida C, Bahia L, Macinko J. O sistema de saúde brasileiro: história, avanços e desafios. Lancet 2011; 377:1719-806.

21. Brasil. Lei no 8.080, de 19 de setembro de 1990. Dispõe sobre as condições para a promoção, proteção e recuperação da saúde, a organização e o funcionamento dos serviços correspondentes e dá outras providências. Diário Oficial da União 1990; 20 set.

22. Rezende CAP. O modelo de gestão do SUS e as ameaças do projeto neoliberal. In: Bravo MIS, organizador. Política de saúde na atual conjuntura: modelos de gestão e agenda para a saúde. 2a Ed. Rio de Janeiro: Rede Sirius/Adufrj-SSind; 2008. p. 25-35.

Recebido em 31/Jul/2013

Versão final reapresentada em 07/Nov/2013

Aprovado em 09/Dez/2013 